\title{
Identifying target processes for microbial electrosynthesis by elementary mode analysis
}

Frauke Kracke ${ }^{1,2}$ and Jens $O$ Krömer ${ }^{1,2^{*}}$

\begin{abstract}
Background: Microbial electrosynthesis and electro fermentation are techniques that aim to optimize microbial production of chemicals and fuels by regulating the cellular redox balance via interaction with electrodes. While the concept is known for decades major knowledge gaps remain, which make it hard to evaluate its biotechnological potential. Here we present an in silico approach to identify beneficial production processes for electro fermentation by elementary mode analysis. Since the fundamentals of electron transport between electrodes and microbes have not been fully uncovered yet, we propose different options and discuss their impact on biomass and product yields.

Results: For the first time 20 different valuable products were screened for their potential to show increased yields during anaerobic electrically enhanced fermentation. Surprisingly we found that an increase in product formation by electrical enhancement is not necessarily dependent on the degree of reduction of the product but rather the metabolic pathway it is derived from. We present a variety of beneficial processes with product yield increases of maximal $36 \%$ in reductive and $84 \%$ in oxidative fermentations and final theoretical product yields up to $100 \%$. This includes compounds that are already produced at industrial scale such as succinic acid, lysine and diaminopentane as well as potential novel bio-commodities such as isoprene, para-hydroxybenzoic acid and para-aminobenzoic acid. Furthermore, it is shown that the way of electron transport has major impact on achievable biomass and product yields. The coupling of electron transport to energy conservation could be identified as crucial for most processes.

Conclusions: This study introduces a powerful tool to determine beneficial substrate and product combinations for electro-fermentation. It also highlights that the maximal yield achievable by bio electrochemical techniques depends strongly on the actual electron transport mechanisms. Therefore it is of great importance to reveal the involved fundamental processes to be able to optimize and advance electro fermentations beyond the level of lab-scale studies.
\end{abstract}

Keywords: Cathode, Electro synthesis, Bio production, Mediator, NAD/NADH, Extracellular electron transport, Electro fermentation, Anaerobic fermentation

\section{Background}

Metabolic redox limitations can be a crucial factor determining the viability of an industrial biotechnology process [1]. It could be shown, that increasing the amount of redox cofactors such as NADH or NADPH available to the microorganisms is an effective way to increase the product yield of reduced products such as propane [2,3] and also of commonly produced feed amino acids, e.g. lysine [4]. One novel and very promising approach to optimize the cellular redox state for

\footnotetext{
* Correspondence: j.kromer@uq.edu.au
'Centre for Microbial Electrosynthesis, The University of Queensland, Level 4,

"Centre for Microbial Electrosynthesis, The University of Queensland, Level 4, Gehrmann Laboratories Building (60), Brisbane, QLD 4072, Australia ${ }^{2}$ Advanced Water Management Centre, The University of Queensland,
Brisbane, OLD, Australia 'Advanced Water Managen
}

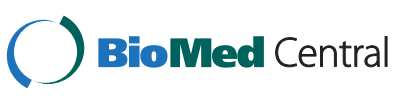

(c) 2014 Kracke and Krömer; licensee BioMed Central. This is an Open Access article distributed under the terms of the Creative Commons Attribution License (http://creativecommons.org/licenses/by/4.0), which permits unrestricted use, distribution, and reproduction in any medium, provided the original work is properly credited. The Creative Commons Public Domain Dedication waiver (http://creativecommons.org/publicdomain/zero/1.0/) applies to the data made available in this article unless otherwise stated. The technique, termed microbial- electrosynthesis or electro fermentation, shows potential to increase the efficiency of microbial production by providing additional electron donors or acceptors to the cells [5,6]. Even though nowadays already discussed as revolutionising future technology, little is known about its true potential as the fundamental processes still remain unclear $[7,8]$.

Before general process design steps can be approached, a better understanding of the overall net benefits of possible target processes is needed [7,9]. These need to feature the production of a higher value carbon-body from a ubiquitous available cheap source by the investment of a reasonable amount of electric energy. While the first 
published electrically enhanced fermentations display in general proof-of-concept-studies, interesting substrate and product combinations still have to be investigated. The biggest challenge to drive microbial electrochemical technologies beyond fundamental studies is the optimisation of the microbial catalyst. Therefore the actual metabolic processes of microbe and electrode interaction need to be unveiled as they will not only decide about the choice of organism but might also play an important role for the achievable process benefit.

The research field of microbial fuel cells studies microbe-electrode interactions for many years. While key reactions could be identified a thorough understanding of the metabolic response to electrical enhancement has not been achieved yet $[10,11]$. The focus of microbial electrosynthesis especially requires more knowledge about cathodic electron transport and extracellular electron transfer (EET) capabilities of model organisms for production such as E. coli.

For anodic EET of electrogens such as Geobacter and Shewanella two main mechanisms are identified: direct electron transfer, which is performed by direct contact between the electrode surface and cellular components of the outer membrane (usually cytochromes), and indirect EET, which includes all forms of electron transfer between electrode and organisms mediated by soluble electron carrier molecules $[12,13]$. First studies on the cathode confirmed the possibility of donating additional electrons to the microbial metabolism by both EET mechanisms and its potential to increase production [5,14-17]. But it was also found that the involved mechanisms for electron uptake differ significantly from the known electron donating mechanisms $[10,18,19]$.

Regardless of the major carbon metabolic pathway the effect of electrical enhancement is typically assumed to result in an increase or decrease of intracellular redox factors such as NADH or NADPH $[8,11]$. Electron transfer towards an anode is assumed to be coupled to energy conservation where the electrode functions as solid final acceptor during respiration [20,21]. However the exact ratio of electrons and protons that are transported remains purely speculative. Furthermore, it is not known by which mechanisms non-metal-respiring organisms might transfer electrons to an anode and whether that transfer promotes ATP generation or not [22]. Even though there is even less information available about cathodic electron transfer there is a general concept proposed that assumes the creation of a proton motive force by intracellular electron consumption, which is available for ATP synthesis $[5,11,23,24]$. In mediated electrically enhanced fermentations of Actinobacillus succinogenes Park and Zeikus observed an electron flow from the cathode into the product succinate [25]. Simultaneously, the electron transfer via the reduced mediator Neutral
Red and the proton-pumping fumarate reductase complex of $A$. succinogenes induced proton translocation and therefore increased ATP synthesis [26]. While the activity of the proton pumping fumarate reductase of $A$. succinogenes is most likely solely responsible for the reported increase in proton flux through the ATPase complex, nowadays the theory about cathodic EET generally assumes that all electrons supplied by EET enter the cytoplasm as negative charge and catalyse intracellular, proton consuming reductions. Simultaneously, the proton consumption would lead to a proton gradient across the inner membrane that drives ATP synthesis [5]. But is this the only possibility? Observed is poor growth in very thin biofilms on cathodes $[15,18]$, which seems to be surprising if cathodic EET could deliver redox power as well as energy (NADH and ATP). So the questions are: Are the protons involved in cathodic electron transfer generally available for ATP generation? And does electron transfer towards an anode always occur by the respiratory chain which thereby creates a proton gradient? What other ways of EET could occur and how would this impact production?

Aim of this work is to present a useful analysis tool, which is able to identify beneficial production processes for microbial electrosynthesis, and at the same time enables insight into the energy conservation possibilities during anaerobic electrically enhanced fermentation. Using in silico approach to calculate the metabolic impact of different electron transport routes during electrically enhanced fermentation enables the evaluation of different mechanisms while current knowledge gaps remain. Pandit et al. recently presented a first computational approach that characterized the general role of bio electrosynthesis in chemical production using a genome scale metabolic model of E. coli [24]. Within their model it is assumed that cathodic electrons enter the metabolism and directly reduce $\mathrm{NAD}^{+}$to NADH. Analogous to the theory discussed before the authors precariously assume the creation of a proton motive force that drives ATP synthesis even though the fumarate reductase of $E$. coli is, unlike the one of $A$. succinogenes, a non-proton-pumping enzyme [27]. Not surprisingly they report an increase of ATP yield caused by electron supply. We regard extracellular electron transport coupled and uncoupled to ATP synthesis and discuss the properties of both options to boost the production of various valuable products. Four different electron transport scenarios for mediated cathodic and anodic EET are described in the following paragraph and are visualised in Figure 1A and B.

\section{Cathode 1 (Cat1)}

Mediator oxidation occurs on outer membrane cytochromes that transfer the electrons into the organism and finally onto NAD. Charge-imbalance creates driving force for 1 proton per electron to enter the cytosol and 


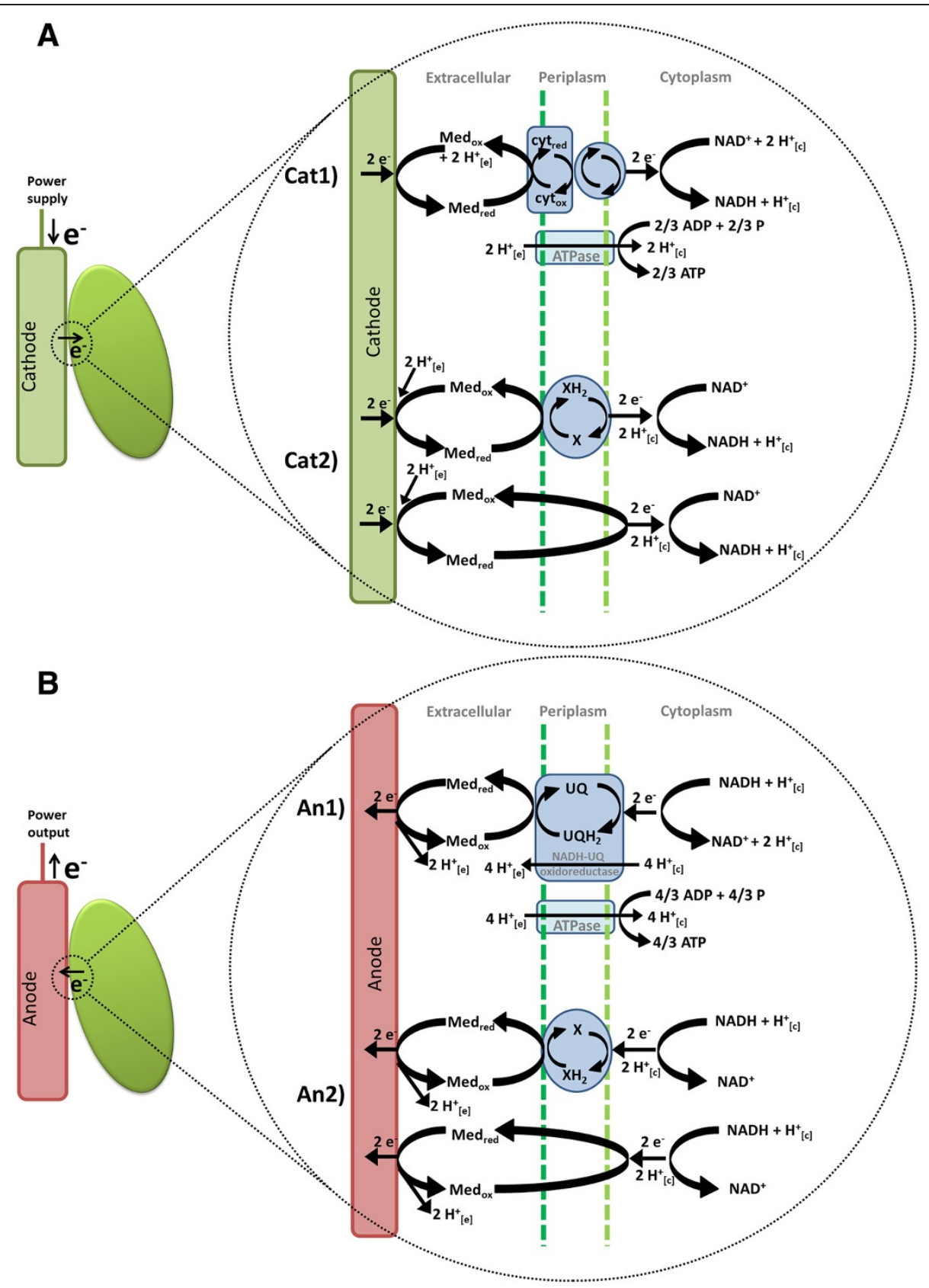

Figure 1 Models for extracellular electron transport mechanisms coupled and uncoupled to energy conservation. (A) Schematic image of two different electron transport mechanisms between cathodes and the microbial metabolism analysed within this study. (at 1) Electron transport via a mediator and a cascade of membrane bound complexes (e.g. cytochromes) with simultaneous ATP generation; Cat2) Direct reduction of NAD to NADH by electrons and protons by membrane bound enzymes (e.g. hydrogenases) or diffusion of the mediator molecule. (B) Two different models for microbial interaction with an anode as electron sink. An1) Electrons from the quinone pool are transferred to the electron mediator by membrane-bound enzymes such as NADH-Ubiquinone oxidoreductase. As these complexes are proton pumping the created gradient can be used for ATP generation. An2) Electrons and protons are transferred simultaneously without creating a membrane potential.

drive ATP-synthase (transport of 3 protons catalyses the generation of one molecule ATP).

\section{Cathode 2 (Cat2)}

The mediator transfers its electrons and protons directly onto NAD without creating a driving force for ATP synthesis. This could happen by diffusion into the cytoplasm or catalyzed by enzymes such as hydrogenases.

\section{Anode 1 (An1)}

Electrons from the Quinone-pool are transferred to the mediator molecule by membrane-bound cytochromes of 
the respiratory chain. Running the electron transport chain via Quinones creates a proton gradient that drives ATP synthesis.

\section{Anode 2 (An2)}

The anode acts like an electron sink by directly accepting electrons from NADH. This could happen either catalyzed by membrane-bound enzymes such as hydrogenases or by diffusion of the mediator into the cytoplasm.

Note that apart from the mediator diffusion model all models could theoretically also happen as direct electron transfer between the electrode surface and the cellular membrane.

\section{Metabolic modelling by elementary mode analysis}

We created core networks of metabolic carbon pathways to determine the effect of electrical enhancement through the different EET ways on production. The tool chosen for the metabolic analysis is elementary mode analysis (EMA), which determines all possible solutions of the metabolic matrix by calculating a unique set of so called elementary flux modes (efms) [28]. Each elementary flux mode pictures the proposed cellular metabolism in steady-state conditions and together all efms span the complete solution space for each network. Within this solution space we can determine maximum yields for certain products and reconstruct carbon fluxes within the network for example to study changes in byproduct formation. The advantage of EMA over other modelling approaches is the calculation of ALL solutions rather than only one best solution (e.g. in flux balance analysis). Thereby we can not only assess theoretical maximum yields for production and biomass formation but are also able to compare all possible metabolic flux distributions, which presents a more holistic view of the impact of each EET model [29].

Elementary mode analysis is based purely on stoichiometry of the reaction equations and steady-state conditions of the organism. Therefore the solution space can be regarded as outer boundaries of the metabolic possibilities. Here we use this effectively to determine the maximum theoretical possible advantage of EET on production. Actual in vivo yields will lie inside the determined solutions space. However they will usually be lower than the theoretical maximum yield and will depend on many factors such as thermodynamics, enzyme kinetics, gene regulation and product toxicity, which are not taken into account here.

The presented metabolic analysis was implemented exemplarily for the central carbon metabolism of Escherichia coli as model organism for industrial biotechnology. Tools for its genetic modification are well established which makes it an attractive host for the production of various compounds. Even though E. coli does not show a comparable electrical activity to Geobacter or Shewanella species, it was shown to be able to exchange electrons with electrodes via soluble mediator molecules [30-32]. Furthermore recent studies report successful transfer of functional molecules from the electron transport chain of Shewanella oneidensis into E. coli and therefore suggest that the microbe could be modified for optimized electron exchange mechanisms $[33,34]$.

\section{Results and discussion}

In the following sections we present calculated carbon yields for the production of biomass and various valuable compounds including carboxylic acids, alcohols and aromatics via electrically enhanced fermentation. The initial idea of microbial electrosynthesis was to start from the fully oxidized substrate $\mathrm{CO}_{2}$ and provide all electrons by an electrode. But a disadvantage of using $\mathrm{CO}_{2}$ as sole substrate is the extremely high electron demand and energy limitation by the strictly anaerobic pathways such as the Wood Ljungdahl pathway. Hence many approaches regard the conversion of organic molecules from waste streams, such as acetate, lactate or glycerol by non-acetogenic organisms as more beneficial [35,36]. Within this work we focus on microbial electrosynthesis from substrates other than $\mathrm{CO}_{2}$, mainly glucose and glycerol, a process which is often referred to as "electro fermentation" [7,37]. Sugar fermentations are dominating in bio-industry and were therefore investigated to determine the potential of electrical enhancement to boost these processes [38]. As a second substrate of interest glycerol was chosen as it represents a cheap $\mathrm{C}$-source often produced as a waste in biodiesel production [39]. Its more reductive state compared to glucose suggests it could result in higher yields when converted into more reduced compounds and require less additional electrons [40]. A current review by Jang et al. summarizes important C2-C6-products and their biological production [38]. We implemented all anaerobic production pathways in our metabolic network and analysed the theoretical yields of each compound under electrical enhancement. Figure 2 shows the metabolic fluxmap of the presented E. coli carbon network including all product pathways. A full list of all maximum product yields, with and without biomass formation as well as the number of computed elementary flux modes for each substrate and product combination can be found in Additional file 1.

\section{Impact of EET mechanism on Biomass yields}

In absence of a final electron acceptor for the respiratory chain the anaerobic formation of biomass is generally limited by the availability of energy and the overproduction of reduced redox equivalents [41]. This becomes clear by studying reaction R1 in Figure 2, which shows the coupling of biomass generation to ATP consumption 


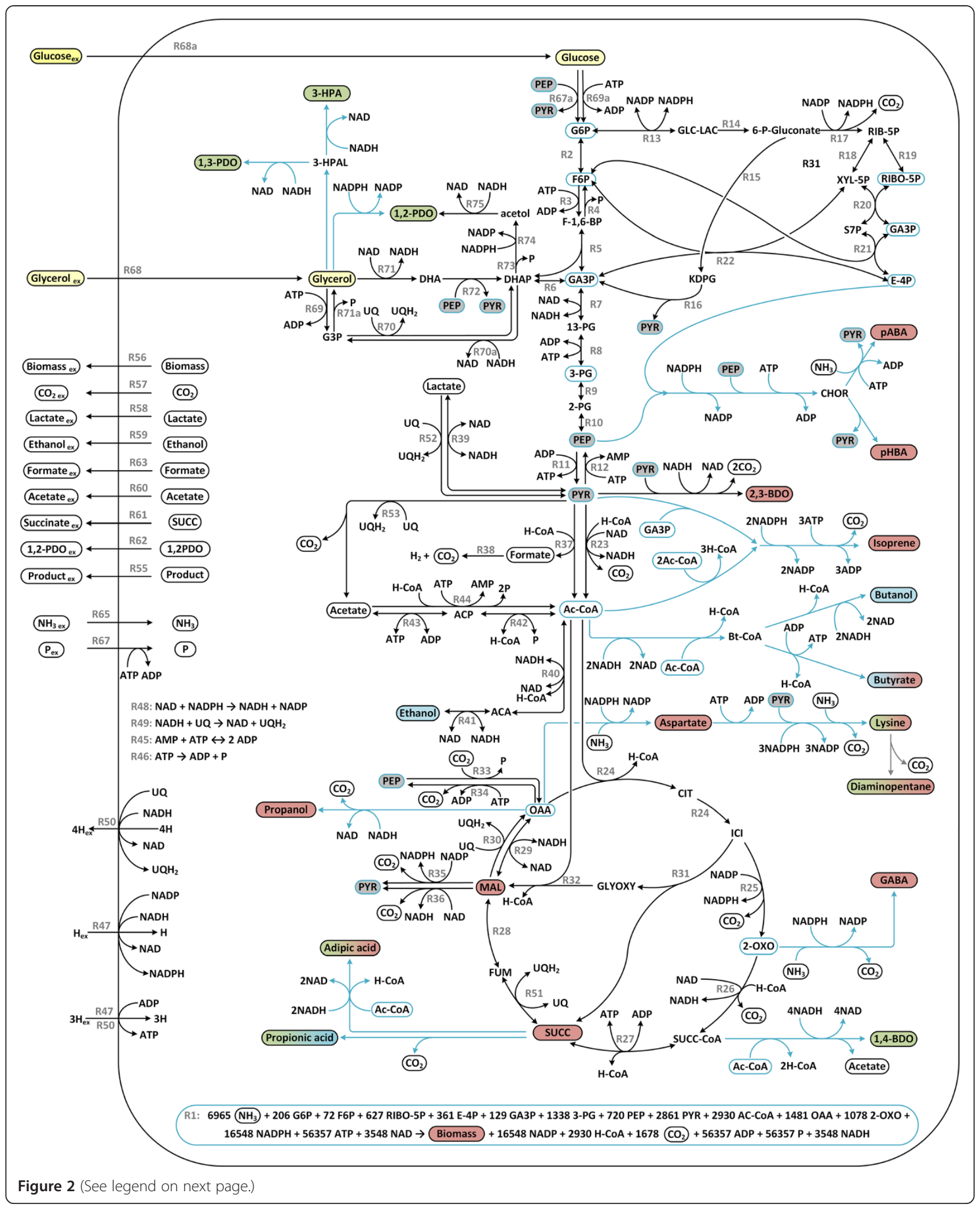


(See figure on previous page.)

Figure 2 Metabolic core network including production pathways. Metabolic fluxmap of the E. coli network including exchange reactions and production pathways. All reactions of the core network are shown with their respective number, RX, as given in Additional file 1. Production pathways are condensed to single reaction steps displayed in light blue. Required precursors for biomass formation (R1) are labelled with blue borderline. The substrates are highlighted in yellow while all target products are coloured according to their most beneficial electron-exchange-option. Increased production by anodes are displayed red, increased product yields by cathodes green while no benefit from either electron transport is shown blue. If a product shows increased yields on different electrodes depending on the substrate a mixed colour pattern was chosen. Key abbreviations are given in the supplementary information.

and $\mathrm{NADH}$ formation. As a result an anode as electron sink increases biomass yields, especially if the energy limitation is lifted by supplying extra ATP $(A n 1)$. On the other hand providing even more electrons through a cathode cannot significantly increase biomass yields. In fact the addition of NADH to the anaerobic network leads to considerably less elementary flux modes as the network has fewer options to distribute carbon fluxes while retaining its redox balance (see efm numbers in Additional file 1).

The maximal carbon yields for biomass production that are achievable with the different electron transport pathways under anaerobic conditions in E. coli are summarized in Figure 3. For the use of glucose as substrate it can be seen that if redox power simultaneously provides additional ATP (Cat1) the biomass yield can be slightly increased, from maximal $26.5 \%$ to $32.5 \%$, while the cathodic model that only supports NADH formation results in a minor yield decrease of about $-0.6 \%$ (Cat 2 ). The network with the anodic model $A n 2$ acting as a pure redox sink is still ATP limited with a maximum achievable biomass yield of about 29.9\%. However the anodic model that supports the creation of a proton motive force, $A n 1$, has the power to enhance biomass production to a maximal yield of $64.1 \%$ which equals an

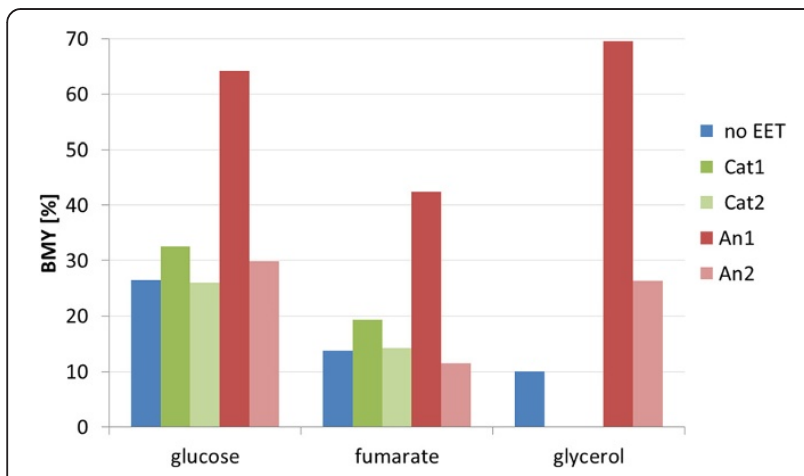

Figure 3 Theoretical maximum biomass yields on different substrates with and without electrical enhancement via different electron-exchange-models. no EET: no electrical enhancement, Cat1: cathodic electron supply coupled to energy conservation; Cat2: cathodic electron supply uncoupled to ATP formation, An 1: anodic redox sink coupled to ATP generation; An2: anodic redox sink uncoupled to energy conservation. increase of about $37.5 \%$ and is close to the theoretical maximal biomass yield under aerobic conditions (71.5\%).

The use of fumarate as substrate was investigated as it is excessively used in literature that studies enhancement of succinate production by EET $[42,43]$. This includes also the studies by Park and Zeikus, which report for the first time the support of growth by electron supply through a cathode $[25,26]$. As discussed in the introduction this might be a unique effect of the enzyme properties of $A$. succinogenes. Activity of its fumarate dehydrogenases complex creates a proton motive force and therefore ATP synthesis while this cannot be translated to other organisms such as E. coli [27]. The maximal biomass yield for $E$. coli grown anaerobically on fumarate was determined to be $13.6 \%$ for non-enhanced conditions while cathodic EET causes an increase to $19.3 \%$ and $14.2 \%$ for Cat1 and Cat2, respectively. To create the amounts of ATP and NADPH needed for biomass formation from fumarate the metabolism is required to produce NADH. Without EET the NADH is mainly produced by the malic enzyme and pyruvate decarboxylase so that in both cases one carbon is "lost" in the form of $\mathrm{CO}_{2}$. NADPH is also created by a malic enzyme under $\mathrm{CO}_{2}$ release (Figure 2). The ATP demand is fulfilled by a combination of running the electron transport chain with $\mathrm{NADH}$ as electron donor and fumarate as final electron acceptor and the acetate producing acetate kinase. This leads to a maximal possible biomass yield of $13.6 \%$ with the main by-products succinate $(55 \%), \mathrm{CO}_{2}$ (19\%) and acetate (13\%). The assumption that cathodic EET results in an increase of available NADH (Cat2) reduces the by-product spectrum to carbon dioxide and succinate only. With fumarate as final electron acceptor the electrons from NADH can enter the first step of the electron transport chain and create a proton motive force, which can drive the highly efficient ATPase. NADPH is created by the membrane bound transhydrogenase driven by proton gradient. This reaction consumes 0.33 ATP equivalents per transhydrogenation (see reaction R47 in Figure 2 and Additional file 1). This results in a maximal biomass yield of $14.2 \%$ for Cat 2 with $6 \% \mathrm{CO}_{2}$ and $80 \%$ succinate as by-products. The extra ATP available in case of Cat1 results in a biomass yield of maximal $19.3 \%$ with $9 \% \mathrm{CO}_{2}$ and $72 \%$ succinate. The high succinate formation in all cases points out that 
fumarate might be an interesting substrate to study electron transport but it is not considered a feasible feedstock for bio-processes due to availability, price and the considerable amount of succinate as a by-product that is to be expected (see above).

Biomass yields calculated for growth on a further reduced substrate such as glycerol cannot be improved by providing additional electrons or protons as the breakdown of glycerol is highly limited by the availability of an electron acceptor. Usually anaerobic growth with glycerol as sole substrate is coupled to the production of hydrogen or 1,3-propanediol as this includes pathway branches that consume NADH created during biomass formation $[40,44]$. If the cellular NADH level is further increased, redox balance can no longer be obtained and growth is inhibited. Still, growth on glycerol with additional electron uptake by a cathode is possible if it is coupled to a production pathway that balances NADH (e.g. propanediol and butanediol see following part of this work). An2 increases the maximal achievable biomass yield on glycerol from $10.0 \%$ to $26.4 \%$. Again $A n 1$ results in a major increase of the max biomass yield up to $69.5 \%$ by providing additional ATP.

The here presented metabolic benefits of increased ATP availability and improved redox balance offered by an anode might be an explanation for the observed thick biofilms on anodic electrodes and poor growth of cathodic cultures $[15,18]$.

\section{Impact of EET mechanism on production}

The decision if microbial electrosynthesis will become an important technique in bio industry will strongly depend on the product yield increase that it can trigger. Therefore it is important to understand the effects of different electron transport routes and energy conservation mechanisms that might happen during electrical enhancement. The degree of reduction (DoR) of a product is often used to describe the electron demand of its production. In fact this is only useful for a direct conversion. The DoR is calculated by the formula given in Table 1 and characterizes a molecule by its oxidative or reductive state.

Figure 4 shows a selection of biotechnologically important substrates and products sorted by their DoR.

Table 1 Formula to calculate the degree of reduction (DoR) for substrates and products (Erickson et al. [45])

\begin{tabular}{lll}
\hline $\mathbf{C}_{\mathbf{a}} \mathbf{H}_{\mathbf{b}} \mathbf{O}_{\mathbf{c}} \mathbf{N}_{\mathbf{d}} \mathbf{S}_{\mathbf{e}} \mathbf{P}_{\mathbf{f}}$ & $\mathbf{C}$ & $\mathbf{+ 4}$ \\
\hline$D \circ R=\frac{4 a+1 b-2 c-3 d+6 e+5 f}{a}$ & $\mathrm{H}$ & +1 \\
& $\mathrm{O}$ & -2 \\
& $\mathrm{~N}$ & -3 \\
& $\mathrm{~S}$ & +6 \\
& $\mathrm{P}$ & +5 \\
\hline
\end{tabular}

Starting from sugars $\left(D_{\text {oR }}\right.$ glucose $\left.=4\right)$ one would expect a benefit from additional electron supply for the production of all compounds with a DoR higher than 4 , such as primary alcohols (e.g. DoR ethanol $=6$ ) or some carboxylic acids (e.g. DoR $R_{\text {butyric acid }}=5$ ). In fact we observe an overall limited predictive power of the DoR as many products with a higher degree of reduction than the substrate show no increased yield with increasing availability of redox equivalents (e.g. ethanol). Contrary we could also find substrate-product-combinations that benefit from extracellular electron supply even though their reductive state is equal (e.g. 3-hydroxy-propionic acid from glucose). Furthermore it was observed that the production of two isomers of the same compound can benefit from opposing redox interference: While the production of 2,3-butanediol is increased in presence of an anode, 1,4-butanediol production benefits from additional electron supply by a cathode (see Additional file 1). Therefore the presented stoichiometric approach is absolutely essential to determine the actual redox balance of a microbial conversion and identify substrate-product-combinations that could benefit from EET.

Figure 2 shows several products that benefit from the presence of a cathode and an anode depending on the used substrate: propionic acid, butyric acid, adipic acid, lysine and diaminopentane. The different response to electrical enhancement can be explained by the use of either glucose or glycerol as carbon-source. All compounds mentioned above are derived from acetyl-CoA or intermediates of the tricarboxylic acid cycle (see Figure 2). To generate these metabolites glucose is broken down by glycolysis where glycerol only enters further downstream. At the end of glycolysis the final metabolite pyruvate is generated with equimolar amounts of NADH and ATP if derived from sugar. With glycerol as the only carbon source 2 mol NADH per mol pyruvate and ATP are created. Therefore some production pathways that re-oxidize only one NADH per pyruvate consumed benefit from an anode on glycerol while a cathode might promote production from glucose. Fully redox-balanced production pathways such as for ethanol or butanol cannot be optimized by electrical enhancement (see Figure 2).

The results of all calculated productions and the effects of the cathodic and anodic electron transport models are summarized in Figure 5 and Additional file 1 and are discussed in the following sections.

\section{Cathodic processes that promote microbial electro reduction}

The elementary mode analysis could identify several carboxylic acids and alcohols that show increased production from glucose and glycerol under extracellular electron supply by a cathode. Propionic acid and adipic acid are both derived from the tricarboxylic acid cycle 


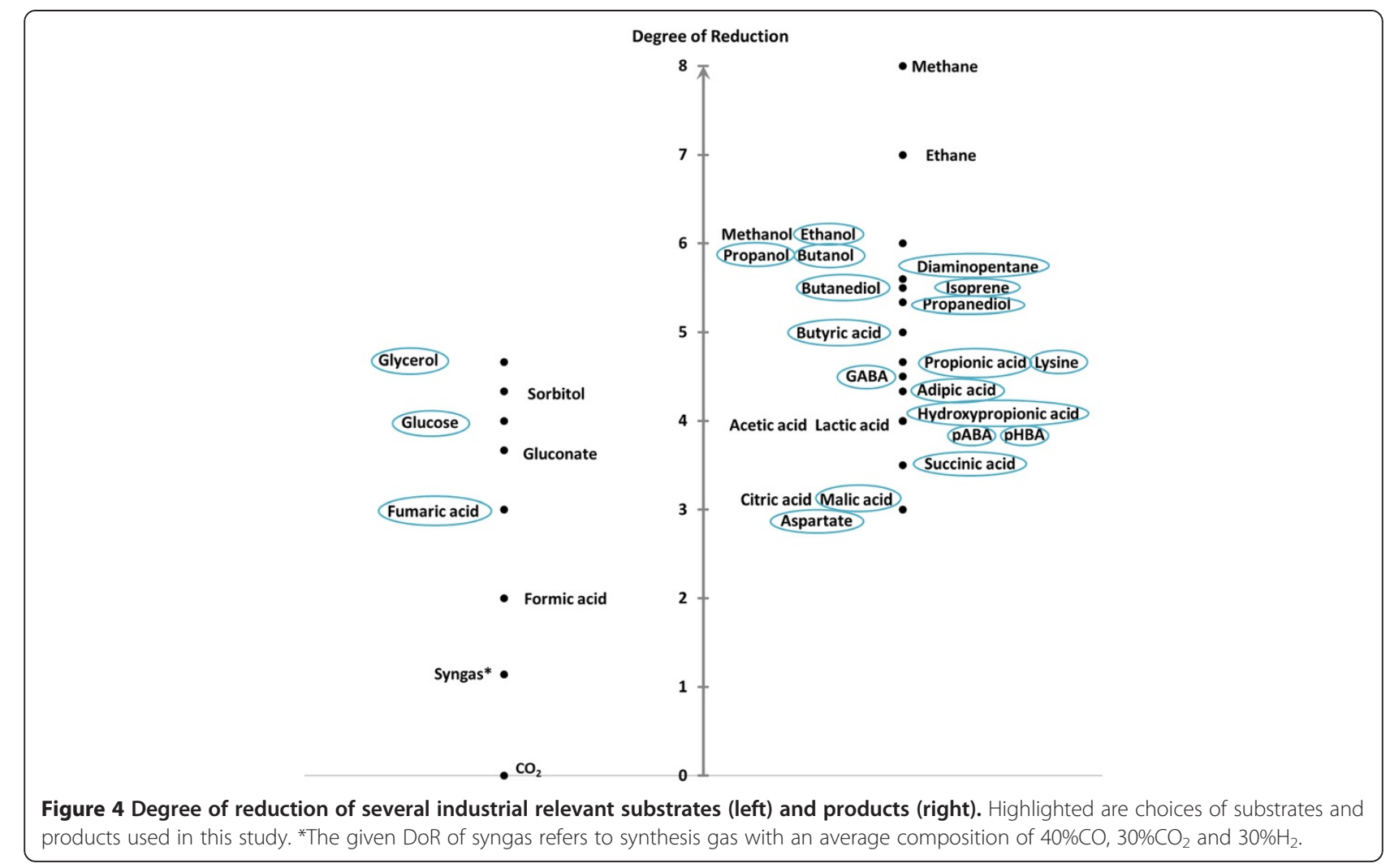

intermediate succinate and show maximum achievable carbon yields of $100 \%$ on a cathode if produced from glucose (see Figure 5A and Additional file 1). Propionic acid is conventionally produced from petro chemicals and has many applications of industrial scale including food additives, perfumes, and pharmaceuticals. Sustainable microbiological production is most promising in natural producers such as Propionibacterium that are able to ferment a variety of carbon sources [46,47]. It could be shown that the use of glycerol is advantageous as its more reduced state compared to hexoses and pentoses benefits the overall metabolic redox state resulting in higher product yields and reduced by-product formation [48]. Accordingly our metabolic analysis determined the maximum achievable yield of propionic acid from glycerol to $100 \%$ (see Additional file 1). We propose that for glucose fermentation this maximum yield can also be achieved if additional electrons are provided by an electrode. The last step of propionic acid production in $E$. coli is the decarboxylation of succinate which results in a "loss" of one carbon atom in $\mathrm{CO}_{2}$. Additional redox power allows the recirculation of $\mathrm{CO}_{2}$ by the oxaloacetate forming phosphoenolpyruvate carboxylase as the increased availability of $\mathrm{NADH}$ enables formation of malate and therefore succinate and PA (see Figure 2). The mechanism by which electrons are fed into the metabolism seems subordinate as for both cathodic models the max yield is $100 \%$. However, comparison of the highest yields that enable simultaneous growth reveals a benefit from scenario Cat1 where the additional ATP input enables a max product yield of $97.7 \%$ with a biomass yield of $2.1 \%$ and $0.2 \% \mathrm{CO}_{2}$ as the only by-products. Electron supply by Cat 2 by contrast results in a maximal growthcoupled product yield of $89.6 \%$ which is not much higher than the non-enhanced production $(85.3 \%)$. A full list of all maximum yields for growth coupled production of each substrate-product-combination is given in Additional file 1 . Also the typical by-products of propionic acid fermentation succinate, $\mathrm{CO}_{2}$ and acetate are detected in more than $85 \%$ of the 1,965 efms of Cat 2 . Whereas none of the 2,840 efms that use Cat1 produces acetate as the influx of electrons and protons results in sufficient ATP production. Emde and Schink reported similar results for in vivo fermentation of Propionibacterium freudenreichii, in which they observed an increased production of propionic acid in presence of reduced mediators while acetate formation was inhibited [14]. According to our calculations this would be an indication towards an EET mechanism as proposed for Cat1 since the shift in product spectrum suggests that the cathode supports an alternative ATP source to acetate production. These results demonstrate that electrical enhancement could be a suitable technique to boost propionic acid production from glucose by reducing by-product formation. But they also 


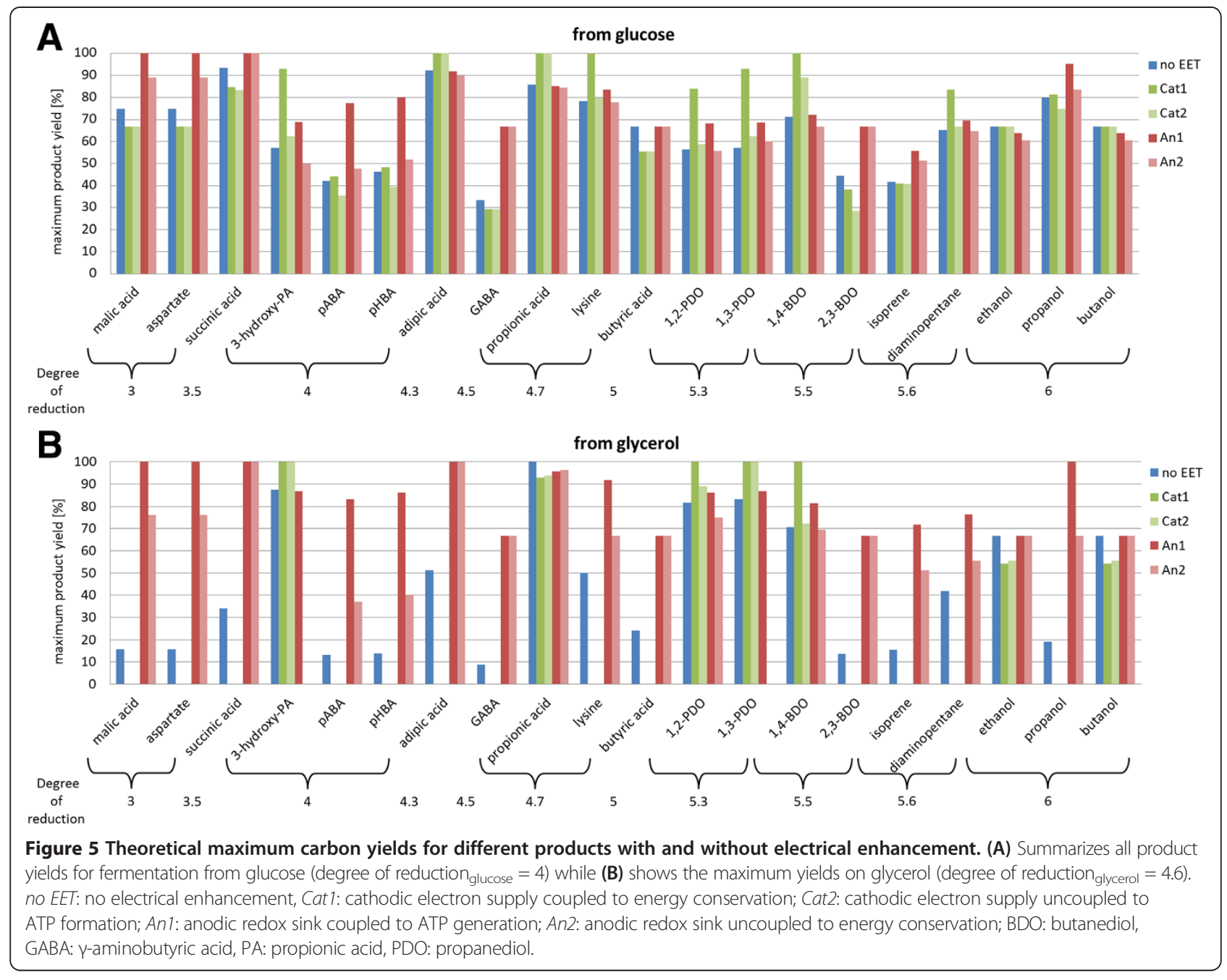

highlight the importance of unveiling the actual connection between extracellular electron transport and energy metabolism for the viability of microbial electrosynthesis.

The second group of products that benefit from additional electron supply by a cathode are compounds derived directly from glycerol or the upper branch of glycolysis such as 3-hydroxypropionic acid, 1,2-propanediol and 1,3-propanediol (Figure 2). 1,2- and 1,3-propanediol (1,2-PDO; 1,3-PDO) are a building blocks for polyesters and even though mostly produced chemically from propylene oxide and propenal, respectively, there are several emerging approaches for their microbiological production. DuPont Tate and Lyle BioProducts have already commercialized several corn-sugar-based 1,3-PDOs (http://www.duponttateandlyle.com). Reported yields of these glucose based fed-batch fermentations with engineered $E$. coli are around 60\% [49-51], which is close to the theoretical maximum product yield on glucose that we computed for a non-enhanced network (57.1\%). Our calculations suggest the maximum yield of this process (which is already on the commercial market!) could be increased up to $92.9 \%$ by electrical enhancement (see Figure 5A and Additional file 1). But the benefit achievable with bioelectrochemical techniques for propanediol production is strongly dependent on the actual EET mechanisms. The product yield during anaerobic glucose fermentation is not only redox but also energy limited. The production of 1,3-PDO from both feedstocks can be summarized with equations 1 and 2 . The usage of glucose requires twice the amount of reducing equivalents and also a high energy phosphate bond $\left(\sim \mathrm{P}_{\mathrm{i}}\right)$ such as ATP or PEP for the phosphorylation of sugar.

$$
\begin{aligned}
& (1) \frac{1}{2} \text { glucose }+\sim \mathrm{P}_{\mathrm{i}}+2 \mathrm{NADH}+2 \mathrm{H}^{+} \rightarrow 1,3-\mathrm{PDO}+ \\
& \mathrm{H}_{2} \mathrm{O}+2 \mathrm{NAD}^{+}+\mathrm{P}_{\mathrm{i}} \\
& \text { (2) glycerol }+\mathrm{NADH}+\mathrm{H}^{+} \rightarrow 1,3-\mathrm{PDO}+\mathrm{H}_{2} \mathrm{O}+\mathrm{NAD}^{+} .
\end{aligned}
$$

Therefore Cat1 which provides simultaneously NADH and ATP causes a significant increase of the maximal 1,3-PDO yield to final $92.9 \%$ for glucose. If Cat 2 would represent the dominant mechanism the product yield 
could only slightly be increased to maximal $62.5 \%$ as the energy limitation would still remain. The production of 1,3-PDO from glycerol also benefits from electron supply by a cathode as seen in Figure 5B. Here both cathodic models result in a maximum yield of $100 \%$, yet the impact on the possible operational options for the network differs significantly for the different EET scenarios. Figure 6 displays the plots of biomass against product yields for each elementary flux mode during production of 1,3-PDO. The transfer of electrons into the cellular metabolism via cytochromes (Cat1) displays for both substrates the most beneficial option (Figure 6C and D). For glucose the product yield is increased significantly and for glycerol not only the maximum achievable yield is improved but also the majority of the cathodic modes features high product and low biomass yields. The production pathway is the most efficient option for the network to maintain cellular redox balance as the internal NADH level is increased. This way cathodic electron supply is automatically coupled to product formation. For the production of PDO from glycerol all modes that take up electrons via Cat1 have product yields above $55 \%$. Moreover $91 \%$ of the total 3,233 modes couple production to biomass formation, which would enable production during the electrochemically enhanced fermentation (Figure 6D). For substrate-product combinations that
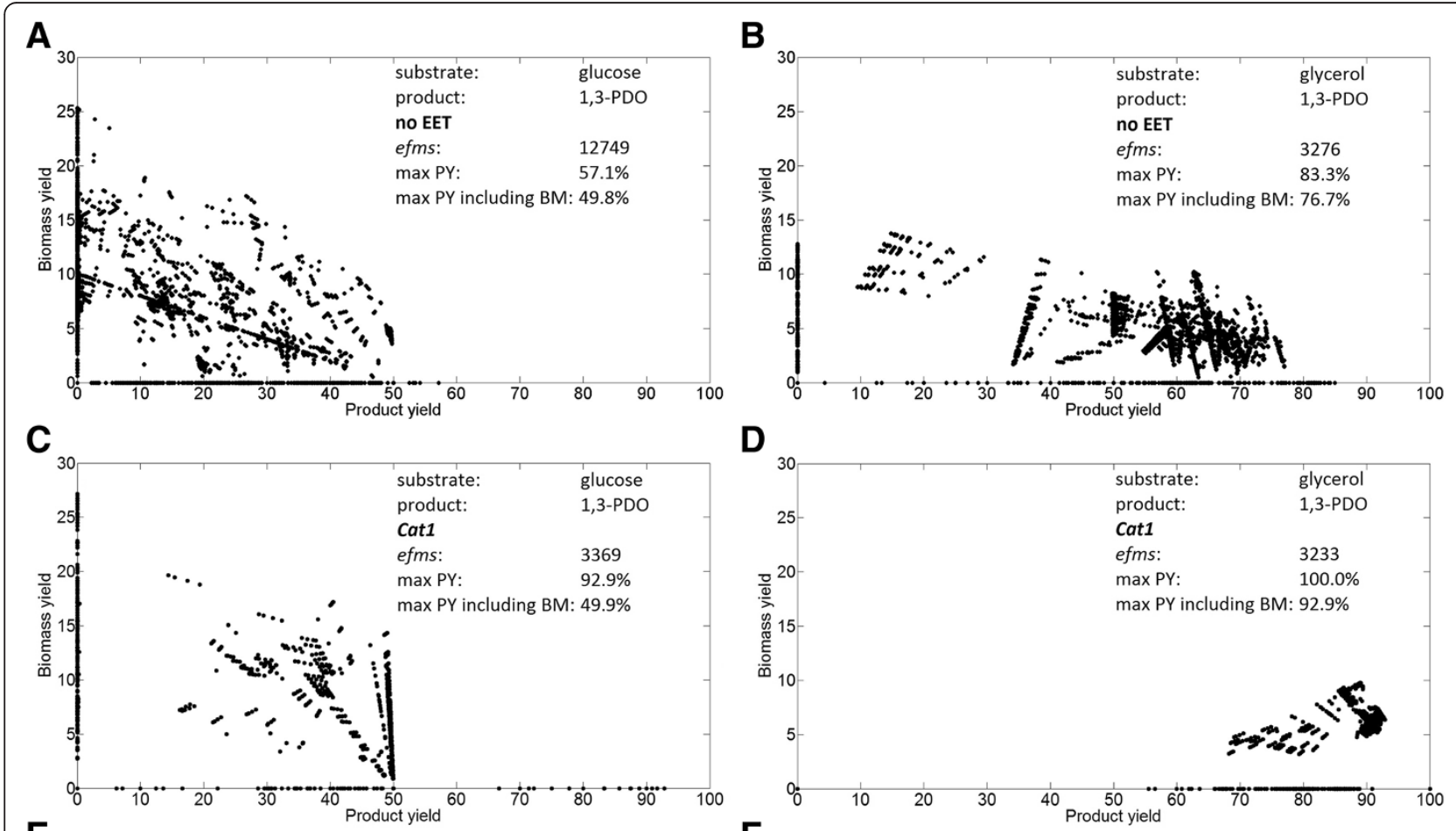

E

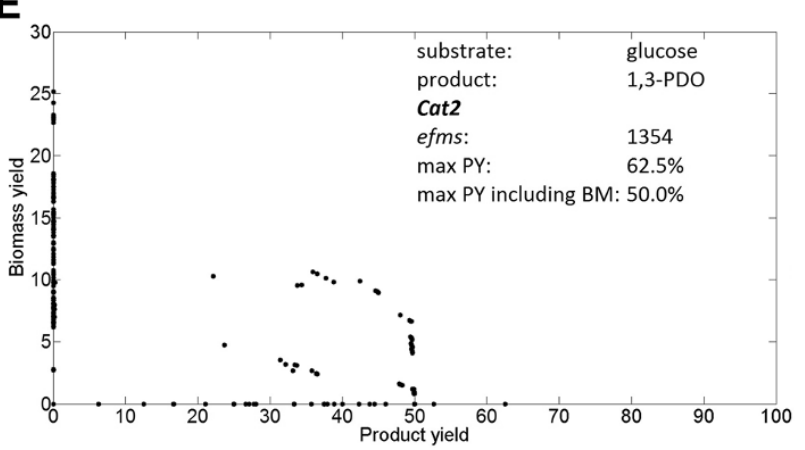

$\mathbf{F}$

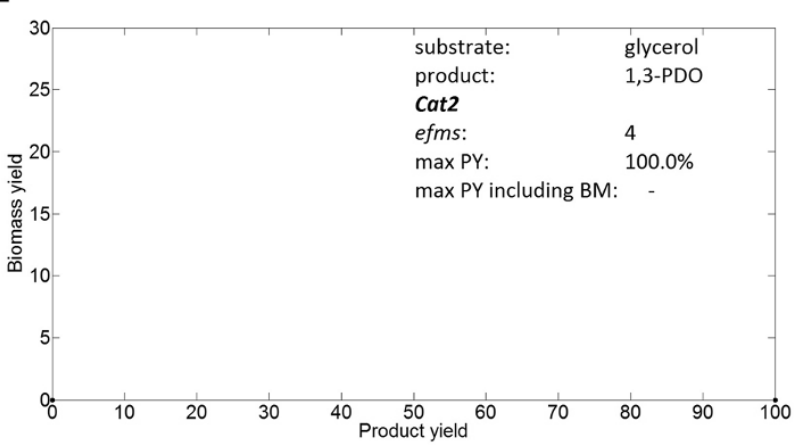

Figure 6 Plots of biomass and product yields of all elementary flux modes for anaerobic 1,3-propanediol production. Each data point in a plot represents the biomass and propanediol yield for a calculated elementary flux mode that uses the respective electron transport model. Graph A, C and E utilize glucose as substrate under different electrical conditions. Graph B, D and F utilize glycerol as substrate under different electrical conditions. Text inserts in each graph summarize the corresponding substrate and product, electron transport model, number of computed elementary flux modes (efms) and maximum theoretical product yields (PY) with and without biomass (BM) production. no EET: no electrical enhancement, Cat1: cathodic electron supply coupled to energy conservation; Cat2: cathodic electron supply uncoupled to ATP formation. 
show this behaviour, electrochemical techniques could offer the possibility to force the metabolism to operate in a desired mode(s) comparable to genetic engineering approaches. But also operational modes that show increased product yields without growth, offer an interesting perspective for microbial electrosynthesis. For the fermentation from glucose it can be seen that all modes above $50 \%$ PDO-yield do not produce any biomass (Cat1) (Figure 6C). This is also the case for both top modes (from glucose and from glycerol) with $100 \%$ product yield. The flux distributions of these particular networks show a carbon flux that could be titled as "true catalysis". The substrate is converted directly into the product by the addition of redox power from the electrode while no by-products are created. To realise "true catalysis" a two-part fermentation strategy could enable successful production by coupling a non-enhanced growth phase to a later electrically supported production phase.

\section{Anodic processes promote microbial electro oxidation}

The use of an anode in bio-electrochemical systems was widely studied in the research of microbial fuel cells, which create electricity as their main product. Substrates are usually mixed waste streams but also glucose and glycerol containing media are used [52-54]. Since the power output of these systems is too small to become relevant on industrial scale any time soon and because of the inherently low value of electricity, the focus shifts towards higher value products $[8,55]$. We identified several valuable compounds that show increased production in presence of an anode (Figure 5 and Additional file 1). It can be seen that without electrical enhancement yields on glycerol are generally a lot lower than on glucose. This is due to the surplus of NADH created during glycerol degradation, which anaerobically becomes limiting if there are no NADH consuming pathway branches such as PDO production [39]. In presence of an anode as electron sink the cellular redox state is optimized and product yields on glycerol can be increased to similar levels as on glucose. Products that benefit from anodic electron transport include glycolysis derived products (isoprene, 2,3-butanediol), products from the tricarboxylic acid cycle (malate, succinate) and its derivatives (propanol, aspartate, $\gamma$-aminobutyric acid) and aromatics from shikimate pathway (para-aminobenzoic acid, para-hydroxybenzoic acid) (see Figure 2). Again the actual EET mechanism is of great importance as the anode that promotes ATP synthesis (An1) triggers significant higher yield increases in many cases (see Figure 5).

2,3-butanediol (2,3-BDO) is an interesting example to study as the production is increased by an anode even though the substrates glucose and glycerol are further oxidized $\left(\mathrm{DoR}_{2,3-\mathrm{BDO}}=5.5, \mathrm{DoR}_{\text {glucose }}=4 ; \mathrm{DoR}_{\text {glycerol }}=4.7\right)$. It also shows reverse behaviour to its isomer 1,4- butanediol that benefits from a cathode, which is due to the different production pathways (see Figure 2 and Additional file 1). 2,3-BDO has applications in the food, pharmaceuticals as well as agrochemical markets and is still produced from fossil fuel feedstocks [38]. But there are several promising approaches for its microbiological production from sugars or glycerol [56]. It is metabolically derived from pyruvate via acetolactate and the recent progress in process optimization suggests bio-2,3-BDO will hit the industrial market soon $[56,57]$. The degradation of glycerol and glucose creates in both cases equimolar ratios of reducing equivalents and pyruvate. Because the production pathway of 2,3-BDO requires only one NADH per two molecules pyruvate an overall surplus of NADH is accumulated. An anode as electron sink can help to optimize NADH/NAD ratios and reduces the formation of by-products such as lactate or ethanol which are otherwise used as electron sink. In this case the transport mechanism of electrons is subordinate as the limitation is purely stoichiometric. Increasing ATP levels cannot improve the maximum yield which is constrained by $\mathrm{CO}_{2}$ formation due to decarboxylation steps during production. Therefore the maximum theoretical yield for 2,3-BDO production on an anode ( $A n 1$ and $A n 2)$ is 66.7\% from glucose and glycerol, respectively (Additional file 1).

However, many compounds are not solely redox limited in their production pathways and therefore the coupling of electron transport to energy conservation is of major importance as was shown before for 1,3-PDO production on a cathode. The here studied production of malic acid, propanol, isoprene, aspartate, para-aminobenzoic acid (pABA) and para-hydroxybenzoic acid (pHBA) on an anode benefits strongly from the proposed mechanism $A n 1$ whereas a pure NADH-redox-sink (An2) results in a significantly smaller yield increase (full table of theoretical yields for all products and EET models is given in Additional file 1). The biggest difference of the two electron transport models is seen for production of the aromatics pHBA and pABA. These are used in sunscreens, dyes, liquid crystal polymers, polyurethanes and food additives and have also the potential to act as building blocks for aromatic polymers [58]. Even though purely synthesised from petro chemicals to date, there is potential for the bio-production of pHBA and pABA as microbes such as $E$. coli produce the aromatics via the shikimate pathway. This pathway requires phosphorylated Co-factors such as NADPH, ATP and PEP and therefore shows major possible flux increases by $A n 1$. pABA yields could theoretically be increased from $42.1 \%$ and $13.1 \%$ to $77.4 \%$ and $83.3 \%$ on glucose and glycerol, respectively. Solely the presence of an anodic electron sink by $A n 1$ causes a shift of the theoretical maximum yield for $\mathrm{pHBA}$ production from $46.3 \%$ to $80.2 \%$ on glucose and $13.9 \%$ to $86.3 \%$ on glycerol (Figure 5). These promising results of redox 
optimisation by electrical interference could offer a new basis for metabolic engineering towards these new products.

\section{Conclusions}

To understand benefits and limitations of microbial electrosynthesis a detailed understanding and analysis of the involved metabolic processes is needed. The presented analysis is the first published approach to methodically screen bio-production processes for their potential benefit from electrical enhancement and could successfully identify 18 target products with possible product yield increases between $7 \%$ and $84 \%$. Even though in vivo yields will usually be lower than the here presented theoretical maximum yields these examples show a great potential of microbial electrochemical techniques to boost anaerobic glycerol and sugar fermentation. Contrary to the assumption reduced compounds such as bio-fuels and alcohols would always require electron input we revealed 12 production processes that show increased product yields on an anode while only 6 of the 20 studied products benefit from additional electron supply. Due to the anoxic fermentation conditions required for an electrically enhanced process intracellular NADH levels are increased, so that in most cases an overall surplus of redox equivalents is accumulated. A benefit from cathodic EET is only seen for production pathways that purely rely on $\mathrm{NADH}$ input (e.g. 1,2-PDO, 1,3-PDO, 3-hydroxypropionic acid) while other products even though further reduced than the substrate are limited by redox surplus and/or energy requirements (e.g. propanol, 2,3-butanediol).

The accumulation of NADH during anaerobic growth could also explain the poor growth performance observed for cathodic cultures. Our analysis shows that the presence of an anode promotes biomass formation while electron supply by a cathode limits the metabolic options of the organism during growth. This limitation could possibly be turned into a benefit by coupling growth to production as explained for the example of the anaerobic conversion of glycerol to 1,3-PDO on a cathode.

The elementary mode analysis does not only identify target processes but also highlights the major importance of electron transport mechanism and its coupling to energy conservation. For the majority of products a crucial dependence of maximum achievable product yield and ATP availability was detected. Therefore it is important to direct the focus of current research in the microbial electrosynthesis community towards fundamentals of electron transport as these are needed to be understood to design processes that approach the full potential of microbial electrochemical techniques.

\section{Methods}

The basic E. coli core model used for the in silico analysis includes: Embden-Meyerhof-Parnas pathway/ glycolysis, glycerol degradation, Entner-Doudoroff pathway, pentose phosphate way, tricarboxylic acid cycle, glyoxylic shunt, anaplerotic reactions, anaerobic fermentation, electron transport chain, import and export reactions and interaction with a soluble electron carrier (see Figures 1 and 2). For the production of industrial relevant products that are not metabolites of the main network, engineered pathway branches for production were implemented. Because oxygen will lead to abiotic current production in most cathodes, we assumed anaerobic conditions as a technical requirement and performed all calculations under anaerobic conditions.

The main network includes 57 metabolites, 75 Reactions (24 reversible) and we calculated up to 215,000 efms per scenario. The full networks can be found in the supplementary information (Additional file 1).

The stoichiometric analysis of metabolic networks was performed based on the elementary mode analysis framework introduced by Schuster et al. [59] The java implemented free software efmtool (version 4.7.1) [60] was used within MATLAB, MathWorks (version R2012a), to compute for each network the elementary flux modes which represent all possible and unique steady-state flux distributions the network could have based on reaction stoichiometry. It does not take regulatory or thermodynamic constraints into account, which means that the theoretical maximum yields are the absolute maximum possible and that in vivo yields will very likely be lower. Nevertheless, it represents the most reliable estimate of the capacity of a network. The operational mode that shows maximum possible carbon flux from substrate into product is referred to as top mode. Maximal theoretical yields for biomass or a certain product are obtained by calculating the carbon balance of all carbon containing substrates entering the network and the carbon containing products leaving the network:

$$
\text { Yield }_{\text {product }}[\%]=\frac{\text { flux }_{\text {product }} \text { carbon }_{\text {product }}}{\text { flux }_{\text {substrate }} \text { Carbon }_{\text {substrate }}} 100 \% .
$$

Where flux product is the reaction rate for products leaving; flux $x_{\text {substrate }}$ the reaction rate for substrate uptake and carbon $_{\text {product }}$ and carbon substrate $_{\text {the number of }}$ carbon atoms in the product and substrate molecules, respectively.

\section{Additional file}

Additional file 1: Metabolic networks and theoretical yields. This file provides detailed information about the studied E. coli carbon networks. The used stoichiometric reactions are given for the core network as well as for all production and electron transport pathways. Furthermore all computed theoretical maximum yields for each substrate product combination and EET scenario are listed including maximum growth-coupled product yields and number of efms. 


\section{Abbreviations}

2,3-BDO: 2,3-Butanediol; DoR: Degree of reduction; EET: Extracellular electron transfer; efm(s): Elementary flux mode(s); pABA: para-Aminobenzoic acid; 1,2-PDO: 1,2-propanediol; 1,3-PDO: 1,3-propanediol; pHBA: Para-Hydroxybenzoic acid

\section{Competing interests}

The authors declare that they have no competing interests.

\section{Authors' contributions}

FK generated the models, performed the analyses and drafted the manuscript. JOK designed the study and guided FK through the modelling. Both authors edited the manuscript. Both authors read and approved the final manuscript.

\section{Acknowledgements}

The authors want to thank The University of Queensland and the Australian Research Council (DE120101549) for financial support.

Received: 10 June 2014 Accepted: 3 December 2014

Published online: 30 December 2014

\section{References}

1. Vickers CE, Klein-Marcuschamer D, Krömer JO: Examining the feasibility of bulk commodity production in Escherichia coli. Biotechnol Lett 2012, 34(4):585-596.

2. Berríos-Rivera SJ, Bennett GN, San K-Y: Metabolic engineering of Escherichia coli: increase of $\mathrm{NADH}$ availability by overexpressing an $\mathrm{NAD}^{+}$-dependent formate dehydrogenase. Metab Eng 2002, 4(3):217-229.

3. Fasan R, Crook NC, Peters MW, Meinhold P, Buelter T, Landwehr M, Cirino PC, Arnold FH: Improved product-per-glucose yields in P450-dependent propane biotransformations using engineered Escherichia coli. Biotechnol Bioeng 2011, 108(3):500-510.

4. Becker J, Zelder O, Häfner S, Schröder H, Wittmann C: From zero to hero-design-based systems metabolic engineering of Corynebacterium glutamicum for I-lysine production. Metab Eng 2011, 13(2):159-168.

5. Lovley DR: Powering microbes with electricity: direct electron transfer from electrodes to microbes. Environ Microbiol Rep 2011, 3(1):27-35.

6. Rabaey K, Rozendal RA: Microbial electrosynthesis - revisiting the electrical route for microbial production. Nat Rev Micro 2010, 8(10):706-716.

7. Rosenbaum MA, Franks AE: Microbial catalysis in bioelectrochemical technologies: status quo, challenges and perspectives. Appl Microbiol Biotechnol 2014, 98(2):509-518.

8. Venkata Mohan S, Velvizhi G, Vamshi Krishna K, Lenin Babu M: Microbial catalyzed electrochemical systems: a bio-factory with multi-facet applications. Bioresour Technol 2014, 165:355-364.

9. Hallenbeck PC: The Future of Biofuels, Biofuels of the Future. In Microbial Technologies in Advanced Biofuels Production. New York: Springer; 2012:261-268.

10. Patil SA, Haegerhaell C, Gortin L: Electron transfer mechanisms between microorganisms and electrodes in bioelectrochemical systems. Bioanal Rev 2012, 4:159-192.

11. Rosenbaum M, Aulenta F, Villano M, Angenent LT: Cathodes as electron donors for microbial metabolism: which extracellular electron transfer mechanisms are involved? Bioresour Technol 2011, 102(1):324-333.

12. Rabaey K, Boon N, Siciliano SD, Verhaege M, Verstraete W: Biofuel cells select for microbial consortia that self-mediate electron transfer. Appl Environ Microbiol 2004, 70(9):5373-5382.

13. Thrash JC, Coates JD: Review: direct and indirect electrical stimulation of microbial metabolism. Environ Sci Technol 2008, 42(11):3921-3931.

14. Emde R, Schink B: Enhanced propionate formation by Propionibacterium freudenreichii subsp. freudenreichii in a three-electrode amperometric culture system. Appl Environ Microbiol 1990, 56(9):2771-2776.

15. Gregory KB, Bond DR, Lovley DR: Graphite electrodes as electron donors for anaerobic respiration. Environ Microbiol 2004, 6(6):596-604.

16. Hongo M, Iwahara M: Electrochemiacal studies on fermentation. Application of electro-energizing method to L-glutamic acid fermentation. Agric Biol Chem 1979, 43(10):2075-2081.

17. Nevin KP, Woodard TL, Franks AE, Summers ZM, Lovley DR: Microbial electrosynthesis: feeding microbes electricity to convert carbon dioxide and water to multicarbon extracellular organic compounds. mBio 2010, 1(2):e00103-10.

18. Dumas C, Basseguy R, Bergel A: Microbial electrocatalysis with Geobacter sulfurreducens biofilm on stainless steel cathodes. Electrochim acta 2008 53(5):2494-2500.

19. Strycharz SM, Glaven RH, Coppi MV, Gannon SM, Perpetua LA, Liu A Nevin KP, Lovley DR: Gene expression and deletion analysis of mechanisms for electron transfer from electrodes to Geobacter sulfurreducens. Bioelectrochemistry 2011, 80(2):142-150.

20. Torres $\mathrm{Cl}$, Marcus AK, Lee HS, Parameswaran P, Krajmalnik-Brown R, Rittmann BE: A kinetic perspective on extracellular electron transfer by anode-respiring bacteria. FEMS Microbiol Rev 2010, 34(1):3-17.

21. Rittmann BE, Torres Cl, Marcus AK: Understanding the Distinguishing Features of a Microbial Fuel Cell as a Biomass-Based Renewable Energy Technology. In Emerging Environmental Technologies. Netherlands: Springer; 2008:1-28.

22. Logan BE: Exoelectrogenic bacteria that power microbial fuel cells. Nat Rev Microbiol 2009, 7(5):375-381.

23. Nevin KP, Hensley SA, Franks AE, Summers ZM, Ou J, Woodard TL, SnoeyenbosWest OL, Lovley DR: Electrosynthesis of organic compounds from carbon dioxide is catalyzed by a diversity of acetogenic microorganisms. Appl Environ Microbiol 2011, 77(9):2882-2886.

24. Pandit AV, Mahadevan R: In silico characterization of microbial electrosynthesis for metabolic engineering of biochemicals. Microb Cell Fact 2011, 10(1):76-76.

25. Park DH, Laivenieks M, Guettler MV, Jain MK, Zeikus JG: Microbial utilization of electrically reduced neutral Red as the sole electron donor for growth and metabolite production. App/ Environ Microbiol 1999, 65(7):2912-2917.

26. Park DH, Zeikus JG: Utilization of electrically reduced neutral Red byActinobacillus succinogenes: physiological function of neutral Red in membrane-driven fumarate reduction and energy conservation. J Bacteriol 1999, 181(8):2403-2410.

27. Iverson TM, Luna-Chavez C, Cecchini G, Rees DC: Structure of the Escherichia coli fumarate reductase respiratory complex. Science 1999, 284(5422):1961-1966.

28. Schuster S, Dandekar T, Fell DA: Detection of elementary flux modes in biochemical networks: a promising tool for pathway analysis and metabolic engineering. Trends Biotechnol 1999, 17(2):53-60.

29. Trinh CT, Wlaschin A, Srienc F: Elementary mode analysis: a useful metabolic pathway analysis tool for characterizing cellular metabolism. Appl Microbiol Biotechnol 2009, 81(5):813-826.

30. McKinlay JB, Zeikus JG: Extracellular iron reduction is mediated in part by neutral red and hydrogenase in Escherichia coli. Appl Environ Microbiol 2004, 70(6):3467-3474.

31. Thompson BG, Gerson DF: Electrochemical control of redox potential in batch cultures of Escherichia coli. Biotechnol Bioeng 1985, 27(10):1512-1515.

32. Xie X-H, Li EL, Tang ZK: Sudden emergence of redox active Escherichia coli phenotype: cyclic voltammetric evidence of the overlapping pathways. Int J Electrochem Sci 2010, 5:1070-1081.

33. Jensen HM, Albers AE, Malley KR, Londer YY, Cohen BE, Helms BA, Weigele $P$, Groves JT, Ajo-Franklin CM: Engineering of a synthetic electron conduit in living cells. Proc Natl Acad Sci U S A 2010, 107(45):19213-19218.

34. TerAvest MA, Zajdel TJ, Ajo-Franklin CM: The Mtr pathway of shewanella oneidensis MR-1 couples substrate utilization to current production in escherichia coli. Chem Electro Chem 2014, 1(11):1874-1879.

35. Desloover J, Arends J, Hennebel T, Rabaey K: Operational and technical considerations for microbial electrosynthesis. Biochem Soc Trans 2012 40(6):1233-1238.

36. Rabaey K, Girguis P, Nielsen LK: Metabolic and practical considerations on microbial electrosynthesis. Curr Opin Biotechnol 2011, 22(3):371-377.

37. Angenent $L T$, Rosenbaum MA: Microbial electrocatalysis to guide biofuel and biochemical bioprocessing. Biofuels 2013, 4(2):131-134.

38. Jang Y-S, Kim B, Shin JH, Choi YJ, Choi S, Song CW, Lee J, Park HG, Lee SY: Bio-based production of C2-C6 platform chemicals. Biotechnol Bioeng 2012, 109(10):2437-2459.

39. Clomburg JM, Gonzalez R: Anaerobic fermentation of glycerol: a platform for renewable fuels and chemicals. Trends Biotechnol 2013, 31(1):20-28.

40. Gonzalez R, Murarka A, Dharmadi Y, Yazdani SS: A new model for the anaerobic fermentation of glycerol in enteric bacteria: Trunk and auxiliary pathways in Escherichia coli. Metab Eng 2008, 10(5):234-245. 
41. Clark DP: The fermentation pathways of Escherichia coli. FEMS Microbiol Lett 1989, 63(3):223-234.

42. Mehta-Kolte MG, Bond DR: Geothrix fermentans secretes Two different redox-active compounds to utilize electron acceptors across a wide range of redox potentials. Appl Environ Microbiol 2012, 78(19):6987-6995.

43. Ross DE, Flynn JM, Baron DB, Gralnick JA, Bond DR: Towards electrosynthesis in shewanella: energetics of reversing the Mtr pathway for reductive metabolism. PLOS ONE 2011, 6(2):e16649.

44. Yazdani SS, Gonzalez R: Anaerobic fermentation of glycerol: a path to economic viability for the biofuels industry. Curr Opin Biotechnol 2007, 18(3):213-219.

45. Erickson LE, Selga SE, Viesturs UE: Application of mass and energy balance regularities to product formation. Biotechnol Bioeng 1978, 20(10):1623-1638.

46. Rodriguez BA, Stowers CC, Pham V, Cox BM: The production of propionic acid, propanol and propylene via sugar fermentation: an industrial perspective on the progress, technical challenges and future outlook. Green Chem 2014.

47. Liu L, Zhu Y, Li J, Wang M, Lee P, Du G, Chen J: Microbial production of propionic acid from propionibacteria: current state, challenges and perspectives. Crit Rev Biotechnol 2012, 32(4):374-381.

48. Zhang A, Yang S-T: Propionic acid production from glycerol by metabolically engineered Propionibacterium acidipropionici. Process Biochem 2009, 44(12):1346-1351.

49. Zeng A-P, Biebl H: Bulk Chemicals from Biotechnology: The Case of 1 , 3-Propanediol Production and the new Trends. In Tools and Applications of Biochemical Engineering Science. Springer; 2002:239-259.

50. Nakamura CE, Whited GM: Metabolic engineering for the microbial production of 1, 3-propanediol. Curr Opin Biotechnol 2003, 14(5):454-459.

51. Laffend LA, Nagarajan V, Nakamura CE: Bioconversion of a Fermentable Carbon Source to 1, 3-Propanediol by a Single Microorganism. Google Patents; 1997.

52. Nimje VR, Chen C-Y, Chen C-C, Chen H-R, Tseng M-J, Jean J-S, Chang Y-F: Glycerol degradation in single-chamber microbial fuel cells. Bioresour Technol 2011, 102(3):2629-2634.

53. Cheng S, Liu H, Logan BE: Increased power generation in a continuous flow MFC with advective flow through the porous anode and reduced electrode spacing. Environ Sci Technol 2006, 40(7):2426-2432.

54. Reiche A, Kirkwood KM: Comparison of Escherichia coli and anaerobic consortia derived from compost as anodic biocatalysts in a glyceroloxidizing microbial fuel cell. Bioresour Technol 2012, 123:318-323.

55. Rabaey K: Bioelectrochemical Systems: From Extracellular Electron Transfer to Biotechnological Application. International Water Assn; 2010.

56. Ji X-J, Huang H, Ouyang P-K: Microbial 2, 3-butanediol production: a state-of-the-art review. Biotechnol Adv 2011, 29(3):351-364.

57. Zeng A-P, Sabra W: Microbial production of diols as platform chemicals: recent progresses. Curr Opin Biotechnol 2011, 22(6):749-757.

58. Koma D, Yamanaka H, Moriyoshi K, Sakai K, Masuda T, Sato Y, Toida K, Ohmoto T: Production of p-Aminobenzoic acid by metabolically engineered Escherichia coli. Biosci Biotechnol Biochem 2014, ahead-of-print:1-8.

59. Schuster S, Hilgetag C: On elementary flux modes in biochemical reaction systems at steady state. J Biol Syst 1994, 2(02):165-182.

60. Terzer M, Stelling J: Large-scale computation of elementary flux modes with bit pattern trees. Bioinformatics 2008, 24(19):2229-2235.

\section{Submit your next manuscript to BioMed Central and take full advantage of:}

- Convenient online submission

- Thorough peer review

- No space constraints or color figure charges

- Immediate publication on acceptance

- Inclusion in PubMed, CAS, Scopus and Google Scholar

- Research which is freely available for redistribution 